ISSN 1991-8631

Original Paper

http://indexmedicus.afro.who.int

\title{
Valeur predictive du PSA dans le diagnostic du cancer de la prostate chez les sujets Beninois
}

\author{
Frédéric LOKO ${ }^{1,2^{*}}$, René HODONOU ${ }^{3,4}$, Romuald AKPAKA ${ }^{4}$, Prince HOUNNASSO ${ }^{3,4}$, \\ Sosthène $\mathrm{ADISSO}^{4,5}$ et César $\mathrm{AKPO}^{3,4}$ \\ ${ }^{1}$ Laboratoire de Recherche en Biologie Appliquée (LARBA/ EPAC/UAC) 01BP 2009 Cotonou, Benin. \\ ${ }^{2}$ Centre d'Information et de Recherche en Santé de la Reproduction (CIRSAR) 03BP2343 Cotonou, Benin. \\ ${ }^{3}$ Clinique Universitaire d'Urologie (CNHU-HKM) $01 B P 386$ Cotonou, Benin. \\ ${ }^{4}$ Faculté des Sciences de la Santé (FSS/UAC) Cotonou, Benin. \\ ${ }^{5}$ Service de Gynécologie-Obstétrique (CNHU-HKM) 01BP386 Cotonou, Benin. \\ *Auteur correspondant, E-mail : lokofrederic@hotmail.com,Tel :00 22995454118
}

\section{RESUME}

Une étude rétrospective et prospective sur un échantillon de 77 patients ayant une tumeur prostatique, a permis de déterminer la valeur prédictive du PSA (prostate specific antigen) dans le diagnostic du cancer de la prostate. La prévalence du cancer de la prostate au sein des patients ayant une tumeur prostatique est de $31 \%$. $100 \%$ des sujets porteurs d'un cancer de la prostate ont un rapport PSAlibre /PSA total $<25 \% ; 79 \%$ des patients porteurs d'un cancer ont une densité du PSA (PSAD) $>0,25 \mathrm{ng} / \mathrm{ml} / \mathrm{cm}^{3}$. La comparaison du PSA total et du toucher rectal (TR) dans le diagnostic du cancer de la prostate a été faite : une sensibilité (50\% et $87 \%$ ), une spécificité $(81 \%$ et $72 \%$ ), une valeur prédictive positive $(54 \%$ et $58 \%$ ), une valeur prédictive négative (72\% et $93 \%$ ) ont été obtenues respectivement pour le TR et le taux de PSA total. Cette étude montre que la détermination du PSA total et libre associée au TR améliore le diagnostic du cancer de la prostate.

(C) 2011 International Formulae Group. All rights reserved.

Mots clés : PSA, cancer de la prostate, hypertrophie bénigne de la prostate, diagnostic.

\section{INTRODUCTION}

L'hypertrophie bénigne de la prostate (HBP), est une tumeur bénigne qui se développe à partir des constituants glandulaires et musculaires de la partie centrale de la prostate. Le cancer de la prostate est une néoformation primitive maligne développée aux dépens des cellules épithéliales de la prostate. C'est le premier cancer urologique et le deuxième cancer chez l'homme après le cancer du poumon en Europe. Son incidence en 2008 aux Etats
Unis est de 198 pour 100000 habitants pour les populations noires et de 63 pour 100000 habitants pour les populations blanches (Vickers et al., 2009). Elle est de 70 pour 100000 habitants en Europe contre 25 pour 100000 habitants en Asie (Namiki et al., 2010).

Les facteurs prédisposant sont l'âge (hommes de plus de 55 ans), les antécédents familiaux, la race (les hommes d'origine africaine sont plus à risque que les Caucasiens, et les Asiatiques), les facteurs 
alimentaires (lipides, produits laitiers pour leur richesse en calcium), les facteurs environnementaux, les hormones (androgènes). D'autres facteurs ont été incriminés : l'obésité, le manque d'exercice physique, le tabagisme, la vasectomie et les infections sexuellement transmissibles.

Le processus cancéreux comprend les trois étapes suivantes: Induction du processus cancéreux par un oncogène affectant 3 clones cellulaires (les clones hormono-sensibles, les clones hormonodépendants, les clones hormonoindépendants), régression (la déplétion androgénique détruit les clones hormonodépendants et hormono-sensibles) et progression (la déplétion androgénique ne retarde pas la croissance des clones hormonorésistants qui se développent). La composition cellulaire devient de plus en plus indifférenciée et hétérogène au fil des doublements successifs ouvrant le champ à des mutations géniques. Ainsi le cancer de la prostate s'installe localement, devient extra corpusculaire et peut se métastaser.

Les signes cliniques ne sont pas spécifiques au cancer de la prostate et peuvent aussi se retrouver dans l'HBP (dysurie, pollakiurie, hématurie, miction par regorgement). Les complications sont mécaniques (rétrécissement urétral, vessie de lutte, diverticules de la vessie, rétention aiguë d'urine, lithiase vésicale, dilatation urétérale, insuffisance rénale) ou infectieuses (infections urinaires, cystite, l'orchiépididymite aiguë).

Le dosage du PSA a permis un diagnostic à un stade localisé, curable par les traitements actuels. Quel que soit le mode de découverte de l'affection, les biopsies prostatiques écho guidées par voie endorectale restent indispensables pour confirmer le diagnostic. La possibilité d'un diagnostic à un stade précoce et curable a permis de réduire la létalité du cancer de la prostate, grâce au recours au dosage du PSA (Prostate Specific Antigen) (Fournier et al., 2004 ; Perracaula et al., 2008 ; Sunami et al., 2009).
Le PSA est une glycoprotéine appartenant au groupe des kallicréines (Hk1, $\mathrm{Hk} 2, \mathrm{Hk} 3$ ) qui constitue le complexe ProPSA (Fournier et al., 2004). La diversité au niveau de la longueur des chaînes polypeptidiques, les différentes possibilités de glycosylations, ont conduit à l'existence d'une dizaine d'isoformes du PSA, dont 3 isoenzymes. Le PSA est une sérine protéase sécrétée en grandes quantités dans le liquide spermatique dont l'activité enzymatique protéolytique est impliquée dans la fluidité de l'éjaculat et faciliterait la migration des spermatozoïdes (Lilja, 2003)

Le marqueur de la maladie prostatique est la chaîne peptidique, et non la chaîne de glycanne (Perracaula et al., 2008; Sunami et al., 2009 ). Ces polypeptides contenus dans différents isoformes ont montré des affinités obligatoires dans leurs structures, ce qui a pour conséquence une élévation de la sensibilité du dosage du PSA dans les maladies prostatiques et aussi une augmentation du nombre de faux positifs (corrélée avec une spécificité abaissée) lors des dépistages du cancer de la prostate (Perracaula et al., 2008; Sunami et al., 2009).

En outre, certains travaux ont rapporté des peptides spécifiques pour différentes formes de PSA, qui sont responsables du cancer de la prostate ou de l'HBP (Perracaula et al., 2008; Sunami et al., 2009).

Le PSA total existe sous deux formes : la fraction libre et la fraction liée à des protéines (alpha-1-anti chymotrypsine [ACT] et alpha-2-macroglobuline [AMG]).

La détermination du seul taux de PSA total pour le diagnostic précoce du cancer de la prostate n'est pas satisfaisante étant donné le pourcentage non négligeable de faux positifs (Gann et al., 2002). En cas de cancer de la prostate, le PSA se trouve en grande partie liée aux protéines plasmatiques et par conséquent, le taux de fraction libre est plus faible. Le taux est plus élevé dans l'HBP. D'où l'intérêt diagnostique différentiel du rapport PSA libre/ PSA total. D'autres paramètres ont été proposés : La densité du 
PSA (PSAD) qui est le rapport entre le taux de PSA et le volume prostatique mesuré par échographie endorectale. Ce paramètre est complémentaire du PSA total pour le diagnostic du cancer de la prostate, mais sa valeur diagnostique est très controversée, du fait de l'erreur liée à l'imprécision de la mesure du volume prostatique (Stephan et al., 2009). Plus récemment, le taux de PSA, rapporté au volume de la zone de transition a été proposé. La détermination du volume de la zone de transition par échographie serait plus précise que le volume total prostatique, ce qui confèrerait une meilleure fiabilité à ce paramètre (Ghafoori et al., 2009). Les auteurs ont également proposé la vélocité du PSA ou PSAV qui est la mesure de l'augmentation moyenne annuelle du taux de PSA au cours de dosages successifs (en général trois sur une période de 18 mois). En effet, il a été montré que contrairement à l'HBP, la majorité des cancers ont une vélocité du PSA supérieure à $0,75 \mathrm{ng} / \mathrm{ml} /$ an (Ghafoori et al., 2009).

La différence de vélocité notée entre le cancer de la prostate et l'HBP est due à l'augmentation de la quantité de PSA sécrété par une unité de masse de la tumeur dans le cas du cancer. Ainsi la quantité de PSA sécrété est de $0,003 \mathrm{ng} / \mathrm{ml} / \mathrm{g}$ en cas d'HBP alors qu'elle est de $0,035 \mathrm{ng} / \mathrm{ml} / \mathrm{g}$ dans le cas $\mathrm{du}$ cancer de la prostate (D'Amico et al., 2004). Cependant, les variations individuelles sont importantes. Certains auteurs ont observé que la vélocité du PSA n'est pas un marqueur très spécifique du cancer de la prostate (Ghafoori et al., 2009). Cependant, l'augmentation de la vélocité du PSA a une valeur pronostique. Les individus souffrant $\mathrm{du}$ cancer de la prostate et présentant une vélocité du PSA supérieure à 2,0 ng/ml dans l'année qui précède le diagnostic, présentent un taux de mortalité plus élevé, en dépit de la prostatectomie (D'Amico et al., 2004).

La prise en compte des dérivés du PSA (PSA libre essentiellement), entre 2,5 et 3 $\mathrm{ng} / \mathrm{ml}$ de PSA total permet de mieux sélectionner les indications de biopsies. Néanmoins, le nombre de biopsies non justifiées augmente au fur et à mesure que le seuil de positivité du PSA diminue. Par conséquent, cet abaissement du seuil est uniquement utile dans les groupes à risque ethnique ou familial (Thompson et al., 2004).

L'insuffisance de la couverture sanitaire, les revenus des populations, insuffisants pour réaliser toutes les explorations prescrites pour le diagnostic des tumeurs prostatiques et une accessibilité géographique aux centres spécialisés limitée, ont suggéré l'étude de la valeur prédictive du PSA dans le diagnostic du cancer de la prostate.

Notre objectif est de comparer la valeur diagnostique du rapport PSA libre / PSA total au taux du PSA total, de comparer ce rapport à d'autres marqueurs proposés (TR et PSAD). Nous pourrons ainsi proposer un outil de diagnostic efficace, susceptible de contribuer à réduire la mortalité due au cancer de la prostate.

\section{MATERIEL ET METHODES Matériel}

Les prélèvements ont été faits à la Clinique Universitaire d'Urologie et d'Andrologie du Centre National Hospitalier et Universitaire Hubert Koutouko Maga (CNHU-HKM) de Cotonou. Ils ont été analysés au laboratoire d'analyses biomédicales du Centre d'Information et de Recherche en Santé de la Reproduction (CIRSAR) (Bénin), affilié au Laboratoire de Recherche en Biologie Appliquée (LARBA/EPAC) de l'Université d'AbomeyCalavi.

\section{Echantillonnage}

Il s'agit d'une étude analytique transversale, rétrospective et prospective, descriptive. La phase rétrospective a couvert la période du $1^{\mathrm{er}}$ janvier au 31 juillet 2008, et la phase prospective du $1^{\text {er }}$ août au 30 novembre 2008.

Notre échantillon est constitué de 250 patients de sexe masculin, âgés de 50 ans et plus, dont 77 sont pris en charge à la Clinique 
Universitaire d'Urologie et d'Andrologie du CNHU-HKM, pour hypertrophie bénigne de la prostate ou cancer de la prostate.

La taille de notre échantillon est supérieure à la taille théorique (206), obtenue par la formule de Schwarz (1978).

Sont inclus dans l'étude, des hommes reçus en consultation pour des bilans systématiques sans signe évocateur d'une pathologie prostatique, des hommes qui présentent des signes évocateurs de la pathologie prostatique et des personnes à risque (essentiellement celles qui ont des antécédents familiaux).

Sont exclus de l'étude, les hommes porteurs d'un système de drainage vésical (sonde urétro vésicale ou cystocathéter suspubien ou cystostomie), les hommes ayant subi une chirurgie prostatique, ou une biopsie prostatique dans les trois semaines qui précèdent le prélèvement sanguin pour les examens biologiques, les hommes souffrant d'une prostatite aiguë ou chronique avec ou sans images de calcification prostatique, et les hommes qui ont eu une éjaculation dans les 24 $\mathrm{h}$ qui précèdent le prélèvement.

\section{Collecte de données}

En clinique, un interrogatoire précise le motif de consultation. Un examen physique a été fait. Nous avons insisté sur l'état général du patient, la présence ou non de globe vésical, et surtout sur les résultats du toucher rectal.

En plus du toucher rectal, le dosage du PSA (total et libre) a été demandé, de même que la réalisation d'une échographie vésicoprostatique et même l'ECBU (examen cytobactériologique de l'urine) en cas de suspicion d'une infection. Généralement ces renseignements suffisent pour affirmer le diagnostic d'HBP ou de cancer de la prostate. En cas de doute, seule la biopsie et l'étude anatomo-pathologique des cellules prélevées nous permettent de faire un diagnostic de certitude. Les données ont été collectées à l'aide d'une fiche de dépouillement individuelle.
Les variables étudiées sont l'âge, les résultats du toucher rectal, l'échographie vésico-prostatique, l'anatomie pathologique (biopsie, pièce opératoire), le PSA total, le PSA libre, le rapport PSA libre/ PSA total, le PSAD.

\section{Dosage du PSA au laboratoire}

Le dosage du PSA total et du PSA libre est basé sur une analyse immunochimique (ELISA) non compétitive sur phase solide, utilisant la technique directe de sandwich. Le réactif CanAg Fujirebio Diagnostics a été utilisé.

\section{Traitement et analyse des données}

La saisie des données a été réalisée à l'aide du logiciel Excel 2007. L'analyse et le traitement des données ont été faits à l'aide du logiciel Epi info Version 3.3.2. Le test de référence retenu est l'examen anatomopathologique.

\section{RESULTATS}

La fréquence de survenue de l'HBP (69\%) est supérieure à celle du cancer $(31 \%)$ (Tableau 1). Chez les patients porteurs d'HBP, la tranche d'âges dominante est 60 69 ans $(40 \%)$ alors qu'elle est de 70 - 79 ans (33\%) chez les patients porteurs de cancer de la prostate (Tableau 2). La moyenne d'âges des patients porteurs d'HBP est 63 ans. Elle est de 66 ans chez les patients porteurs de cancer de la prostate. 60 patients sur 77 ne présentaient pas d'antécédents urologiques. Néanmoins 17 patients avaient des antécédents d'urétrite (gonococcique ou chlamydienne) (Tableau 3). La moyenne de la valeur du PSA total chez les patients porteurs de cancer de la prostate est 18 fois supérieure à celle des patients porteurs d'HBP (Tableau 4). $89 \%$ des patients ayant le cancer de la prostate ont un ratio PSA libre / PSA total $<15 \%$; aucun d'eux n'a un ratio $>25 \%$. En ce qui concerne les patients ayant l'HBP, 65\% ont un ratio > 25\% (Tableau 5). $79 \%$ des patients qui présentent le cancer de la prostate, ont un PSAD > $0,25 \mathrm{ng} / \mathrm{ml} / \mathrm{cm}^{3}$ contre $21 \%$ 
qui ont un PSAD $<0,15 \mathrm{ng} / \mathrm{ml} / \mathrm{cm}^{3}$. En ce qui concerne les patients présentant l'HBP, 75\% ont un PSAD $<0,15 \mathrm{ng} / \mathrm{ml} / \mathrm{cm}^{3}$ alors que $19 \%$ ont un PSAD $>0,25 \mathrm{ng} / \mathrm{ml} / \mathrm{cm}^{3}$ (Tableau 6)

Une étude comparative de l'efficacité diagnostique du TR et du taux de PSA total dans le diagnostic du cancer de la prostate a été faite: une sensibilité $(50 \%$ et $87 \%)$, une spécificité (81et $72 \%$ ), une valeur prédictive positive ( $54 \%$ et $58 \%$ ), une valeur prédictive négative $(72 \%$ et $93 \%)$ ont été obtenues respectivement pour le TR et le taux de PSA total (pour un cut off de $10 \mathrm{ng} / \mathrm{ml}$ pour le PSA total) (Tableau 7).

Tableau 1: Pathologies prostatiques et leurs fréquences relatives.

\begin{tabular}{lccccc}
\hline & Total & \multicolumn{2}{c}{ HBP } & \multicolumn{2}{c}{ Cancer } \\
\cline { 3 - 6 } & & $\mathbf{N}$ & $\boldsymbol{\%}$ & $\mathbf{N}$ & $\boldsymbol{\%}$ \\
\hline Notre série & 77 & 53 & 69 & 24 & 31 \\
\hline
\end{tabular}

Tableau 2 : Répartition des patients porteurs de tumeurs prostatiques selon l'âge.

\begin{tabular}{lcccc}
\hline & Tumeur & \multicolumn{2}{c}{ HBP } & \multicolumn{2}{c}{ Cancer } \\
\cline { 2 - 5 } Age (ans) & $\mathbf{N}$ & \% & N & $\%$ \\
\hline $50-59$ & & & & \\
$60-69$ & 19 & 35 & 7 & 29 \\
$70-79$ & 21 & 40 & 7 & 29 \\
$80-89$ & 10 & 20 & 8 & 33 \\
$\geq 90$ & 2 & 3 & 2 & 9 \\
Total & 1 & 2 & 0 & 0 \\
\hline
\end{tabular}

Tableau 3: Antécédents d'urétrites et tumeurs prostatiques (Nombre de cas).

\begin{tabular}{lcc}
\hline Urétrite & Oui & Non \\
\hline Cancer & 1 & 23 \\
HBP & 16 & 37 \\
\hline Total & 17 & 60 \\
\hline $\mathrm{X}^{2}=5,08$ & $\mathrm{P}=0,02$ &
\end{tabular}

Tableau 4: Valeur moyenne du PSA (ng/ ml) selon le type de tumeur prostatique.

\begin{tabular}{lcc}
\hline Tumeur & HBP & Cancer \\
\hline Nombre & 53 & 24 \\
Valeur moyenne & 12,81 & 241,7 \\
\hline
\end{tabular}


Tableau 5: Rapport PSA libre / PSA total et tumeurs prostatiques.

\begin{tabular}{lcccc}
\hline Rapport PSA 1/PSA t & \multicolumn{2}{c}{ Cancer } & \multicolumn{2}{c}{ HBP } \\
\cline { 2 - 5 } & Nb & \% & Nb & \% \\
\hline & 16 & 89 & 6 & 21 \\
$15 \%$ & 2 & 11 & 4 & 14 \\
$>25 \%$ & 0 & 0 & 19 & 65 \\
Total & 18 & 100 & 29 & 100 \\
& & & & \\
\hline
\end{tabular}

Tableau 6 : PSAD et tumeurs prostatiques.

\begin{tabular}{lcccc}
\hline PSAD $\left(\mathbf{n g} / \mathbf{m l}^{\prime} \mathbf{c m}^{\mathbf{3}}\right)$ & \multicolumn{2}{c}{ Cancer } & \multicolumn{2}{c}{ HBP } \\
\cline { 2 - 6 } & Nb & \% & Nb & \% \\
\hline$<0,15$ & 5 & 21 & 40 & 75 \\
$0,15-0,25$ & 0 & 0 & 3 & 6 \\
$>0,25$ & 19 & 79 & 10 & 19 \\
\hline Total & 24 & 100 & 53 & 100 \\
\hline
\end{tabular}

Tableau 7: Performances du TR et du dosage du PSA total.

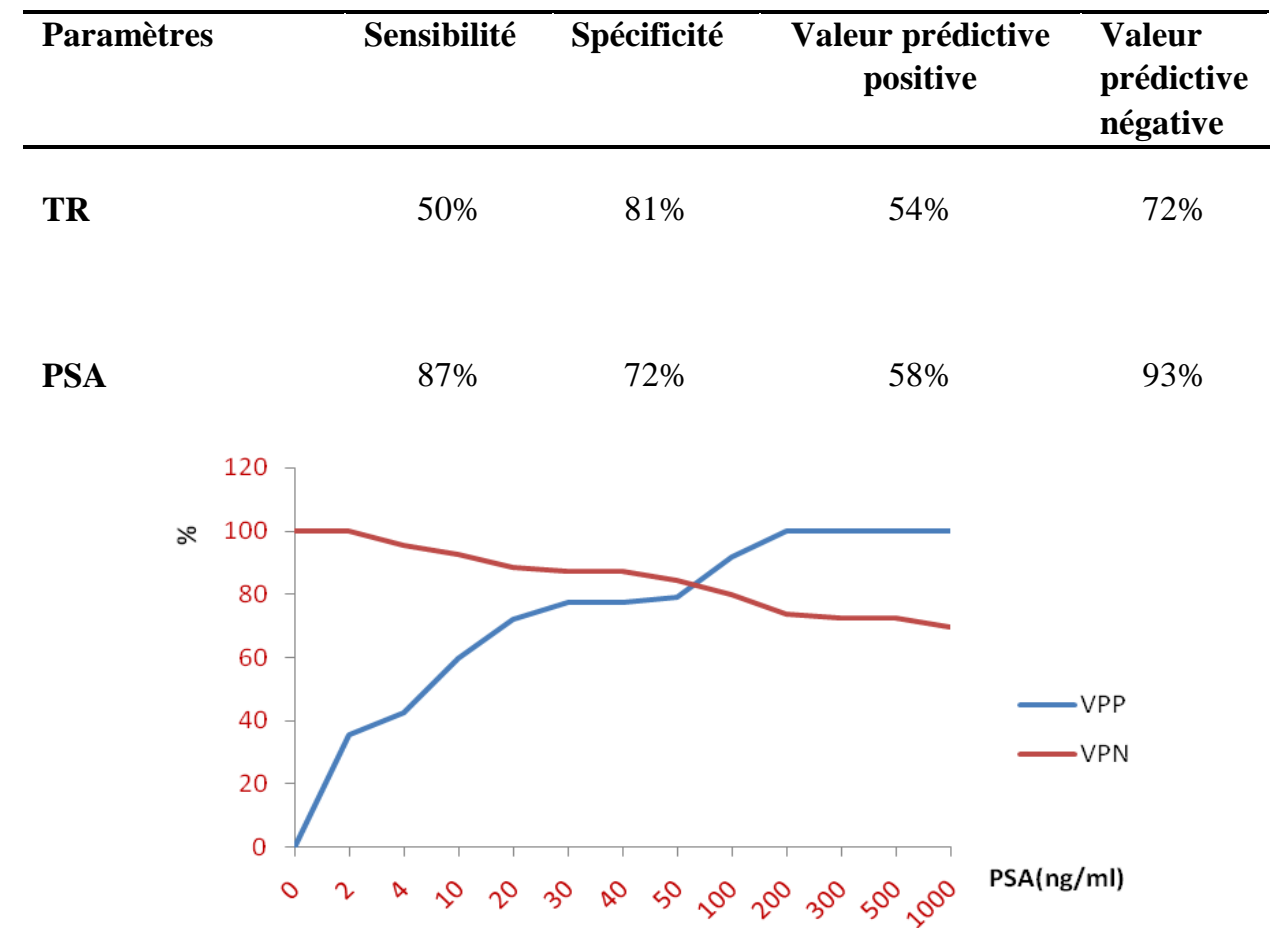

Figure 1: Courbe illustrant la valeur prédictive positive et la valeur prédictive négative du PSA pour le diagnostic de cancer.

$\mathrm{VPP}=$ Valeur prédictive positive $; \mathrm{VPN}=$ Valeur prédictive négative $;$ Lorsque le taux de PSA total varie de 0 à $1000 \mathrm{ng} / \mathrm{ml}$ : - la VPP varie de 0 à 100\%. ; la VPN varie de 100 à $70 \%$. 


\section{DISCUSSION}

La prévalence du cancer de la prostate (31\%), comparée à l'hypertrophie bénigne de la prostate, obtenue dans notre étude, est supérieure à celle obtenue par Namiki et al. (2010), dans une population de patients pris en charge pour l'HBP ou le cancer de la prostate dans un contexte européen. Ces données tendent à prouver que le cancer de la prostate est plus fréquent chez les Noirs que chez les individus de race blanche. On évoque également l'accès à des méthodes diagnostiques plus performantes. Ainsi, l'échographie vésico-prostatique, la sensibilité du dosage du PSA total, l'introduction du dosage du PSA libre, et la précision du toucher rectal dans un service spécialisé ont apporté une meilleure appréciation des tumeurs prostatiques. Selon Namiki et al. (2010). le vieillissement de la population et la performance des moyens de dépistage et de diagnostic permettent d'expliquer l'augmentation de la fréquence du cancer prostatique, avec une incidence de 38 pour 100.000 habitants/an en France.

La moyenne d'âges (66 ans), des patients porteurs de cancer de la prostate dans notre échantillon est comparable à celle obtenue par Thompson et al. (2004) (68 ans) et confirme bien que le cancer de la prostate est une maladie de la vieillesse.

La majorité des patients ayant une tumeur prostatique n'ont pas d'antécédents urologiques. Le taux élevé d'urétrites (chlamydienne ou gonococcique) chez les patients porteurs d'HBP, pose le problème de la prévalence des urétrites dans la survenue ou comme conséquence de l'HBP; d'où la nécessité de bien traiter toute urétrite infectieuse.

$\mathrm{Au}$ seuil de $10 \mathrm{ng} / \mathrm{ml}$ pour le PSA total dans notre étude, et de $4 \mathrm{ng} / \mathrm{ml}$ pour GANN et al. (2002), les ratios (cancer-faux positifs) sont de 1,4 et 1,03 respectivement. Lorsqu'on associe la détermination du PSA libre, ces ratios augmentent respectivement à 1,60 et 1,19 . Les meilleurs ratios obtenus dans notre étude peuvent être reliés au seuil plus élevé du
PSA total $(10 \mathrm{ng} / \mathrm{ml})$ retenu. Le recours à la détermination du PSA libre permet de diminuer le nombre de faux positifs (Pinsky et al., 2005) et d'améliorer la contribution du laboratoire de biologie au diagnostic du cancer de la prostate.

Dans notre étude, la sensibilité du PSA (définie au seuil de $10 \mathrm{ng} / \mathrm{ml}$ ) est la probabilité que le taux de PSA total chez les sujets atteints du cancer de la prostate soit supérieur à ce seuil. Dans notre étude, la sensibilité du PSA total est comparable à celle de Mistry et al. (2003) (72\% au seuil de $4 \mathrm{ng} / \mathrm{ml}$ ) et supérieure à celle de GANN et al. (2002) qui ont rapporté une sensibilité de 31\% au seuil de $4 \mathrm{ng} / \mathrm{mL}$.

La spécificité du PSA, est la probabilité que le PSA soit inférieur à 10 $\mathrm{ng} / \mathrm{ml}$ chez les sujets non atteints du cancer de la prostate. Cette spécificité (94\% dans notre étude) est comparable à celle obtenue par GANN et al. (2002) (95\%) et Gregorio et al. (2007) (91\%).

La valeur prédictive positive est la probabilité qu'un diagnostic de cancer de la prostate soit posé chaque fois que le PSA > 10 $\mathrm{ng} / \mathrm{ml}$. Cette probabilité, dans notre étude, est de $58 \%$. Ce résultat est supérieur à celui de Gregorio et al. (2007) qui ont obtenu un taux de $20 \%$ pour une valeur seuil du PSA à 4 $\mathrm{ng} / \mathrm{ml}$, et GANN et al. (2002) qui ont déterminé une valeur de $11 \%$.

Une étude américaine PCPT (Prostate Cancer Prévention Trial) (Pinsky et al., 2005) a comparé les effets d'un inhibiteur de la 5alpha réductase (Finasteride, Proscar $\AA$ ) au placebo. Dans le groupe placebo, 9.459 hommes d'âge moyen de 62 ans, sans cancer de prostate et un PSA $<3 \mathrm{ng} / \mathrm{ml}$, ont été suivis pendant 7 ans. Ceux qui ont présenté un toucher rectal anormal ou un PSA $>4 \mathrm{ng} / \mathrm{ml}$ (4.692 patients) ont subi une biopsie qui a permis de dépister 571 cancers. On obtient ainsi un taux de détection de $12,2 \%$ une valeur prédictive positive de $41,2 \%$ pour le PSA total inférieure à celle obtenue dans notre étude. 
La valeur prédictive négative (VPN) est la probabilité que le diagnostic du cancer de la prostate ne soit pas posé chaque fois que le PSA $<10 \mathrm{ng} / \mathrm{ml}$. Dans notre étude, la VPN est de $93 \%$. La courbe illustrant la variation de la valeur prédictive positive et la valeur prédictive négative en fonction du taux du PSA total montre qu'à partir d'une valeur de 200 ng/ml de PSA total, le diagnostic de cancer devient une certitude. Ces taux de PSA élevés ne sont pas seulement corrélés au volume prostatique mais aussi à la présence de métastases (Mistry et al., 2003 ).

$79 \%$ des patients chez qui le diagnostic du cancer de la prostate est retenu, ont un PSAD >0,25. 100\% des sujets porteurs d'un cancer de la prostate ont un rapport PSAlibre /PSA total $<25 \%$. La valeur diagnostique du PSAD est donc inférieure à celle du rapport PSA libre /PSA total. Par ailleurs, Catalona et al. (2000) ont évoqué l'imprécision du volume prostatique à l'échographie. Ces deux arguments pourraient expliquer la préférence accordée en pratique courante à la détermination du rapport PSA libre/PSA total.

La sensibilité et les valeurs prédictives positive et négative du PSA sont supérieures à celles du TR. La spécificité du TR est supérieure à celle du PSA.

Fournier et al. (2004) ont trouvé également une sensibilité du PSA supérieure à celle du TR. Par contre la spécificité du PSA était supérieure à celle du TR. En conséquence, une association du dosage du PSA et du TR améliore le diagnostic du cancer de la prostate.

La spécificité modérée du dosage du PSA sérique est imputable à l'existence d'une variété d'isoformes qu'on retrouve aussi bien chez les sujets sains que dans les cas d'hypertrophie bénigne de la prostate et du cancer de la prostate. Cette variabilité moléculaire est due à une hétérogénéité structurale du PSA qui caractérise la plupart des glycoprotéines (Vickers et al., 2009).

Au cours des transformations malignes, cette micro hétérogénéité consiste en une modification de la structure de la fraction protéique (Perracaula, 2008) à laquelle s'ajoute une altération de la glycosylation de la copule glycannique dans le sens d'une augmentation des chaînes complexes et d'une diminution des chaînes oligo-mannosidiques (Reis, 2010).

En somme, à l'instar de tous les marqueurs tumoraux, la détermination du PSA total a une valeur diagnostique modérée liée essentiellement à sa spécificité modérée. Même si le diagnostic du cancer de la prostate est pratiquement certain aux valeurs élevées de PSA total, le dosage du PSA libre et surtout le rapport PSA libre/PSA total ont une bonne valeur diagnostique plus élevée que celle du PSAD pour les taux de PSA total modérément élevés. Par ailleurs, au regard de la VPP modérément élevée du PSA total, nous recommandons vivement que ces tests soient complétés par le TR. L'association de ces tests permet de poser un excellent diagnostic ; en cas de doute, le diagnostic de certitude est réservé à la biopsie (méthode de référence).

$\mathrm{Au}$ plan des perspectives, il faut préciser que certains auteurs (Abbott et al., 2010) ont signalé une grande spécificité de la copule glycannique du PSA au cours du cancer de la prostate, lui conférant un rôle de biomarqueur plus spécifique, exploitable dans la mise au point de méthodes de diagnostic biologique du cancer de la prostate plus performantes que le ratio PSA libre/PSA total.

\section{REMERCIEMENTS}

Nous tenons à exprimer nos sincères remerciements aux autorités du Centre d'Information et de Recherche en Santé de la Reproduction pour le soutien matériel qu'elles nous ont apporté au cours de nos travaux de recherche.

\section{REFERENCES}

Abbott KL, Lim JM, Wells L, Benigno BB, Mc Donald JF, Pierce M. 2010. Identification of candidate biomarkers with cancers specific glycosylation in the tissue and serum of endometrioid ovarian 
cancer patients by glycoproteomic analysis. Proteomics, 10: 470-481.

D'Amico A, Chen M, Roehl K, Catalona W. 2004. Preoperative velocity and tisk death from prostate cancer after radical prostatectomy. New Eng. J. Med., 351(2): 125-135.

Catalona WJ, Southwick PC, Slawin KM, Partin AW, Brawer MK, FlaniganRC. 2000. Comparison of percent free PSA, PSA density, and age-specific PSA cut offs for prostate cancer detection and staging. Urology, 56: 255-566.

Fournier G, Valeri A, Mangin P, Cussenot O. 2004. Cancer de la prostate. Diagnostic et bilan d'extension. Encyclopédie médicochirurgicale, 18: 560.

Gann PH, Ma J, Catalona WJ, Stampfer MJ. 2002. Strategies combining total and percent free prostate specific antigen for detecting prostate cancer: a prospective evaluation. J. Urol., 167: 2427-2434.

Ghafoori M, Varedi P, Hosseini SJ, Asgari M, Shakiba M. 2009. Value of prostatespecific antigen and prostate-specific antigen density in detection of prostate cancer in an Iranian population of men. Urol. J., 6(3): 182-188.

Gregorio EP, Grando JP, Saqueti EE, Almeida SH, Moreira HA, Rodrigues MA. 2007. Comparison between PSA density, free PSA percentage and PSA density in the transition zone in the detection of prostate cancer in patients with serum PSA between 4 and $10 \mathrm{ng} / \mathrm{ml}$. Int.Braz.J.Urol., 33(2): 151-160.

Laurent G. 2003. Cancer de la prostate et nutrition. J. Urol., 4: 144- 147.

Lilja H. 2003. Biology of prostate-specific antigen. Urology, 62(5): 27-33.

Namiki M, Akaza H, Lee SE, Song JM, Umbass R, Zhou L, Lee BC, Cheng C, Chung MK, Fukagai T, Hinotsu S, Horie S. 2010.Prostate cancer Working Group report. Jpn. J. Clin. Oncol., 1: 170-175.

Peracaula R, Barrabés S, Sarrats A, Rudd PM, de Llorens R. 2008. Altered glycosylation in tumours focused to cancer. Diagnosis Dis. Marker, 25: 207-218.

Pinsky PF, Andriole GL, Kramer BS, Hayes RB, Prorok PC, Gohagan JK. 2005. Prostate, Lung, Colorectal and Ovarian Project Team. Prostate biopsy following a positive screen in the prostate, lung, colorectal and ovarian cancer screening trial. J. Urol., 173: 746-750.

Reis CA, Osoriott, Silval Gomes C, David L. 2010. Alteration in glycosylation as biomarkers for cancers detection. J.Clin. Pathol., 63: 322-329.

Schwarz G. 1978. Estimating the dimension of a model. Ann. Statist., 6: 461-464.

Stephan C, Stroebel G, Heinau M, Lenz A, Roemer A, Lein M, Schnorr D, Loening SA, Jung K. 2005. The ratio of prostatespecific antigen (PSA) to prostate volume (PSA density) as a parameter to improve the detection of prostate carcinoma in PSA values in the range of $<4 \mathrm{ng} / \mathrm{ml}$. Cancer, 104(5): 993-1003.

Sunami E, Shinozaki M, Higano CS, Wollman R, Dorff TB, Tucker SJ, Martinez SR, Singer FR, Hoon DS. 2009. Multimarker Circulating DNA Assay for Assessing Blood of Prostate Cancer Patient. Clin. Chem., 74: 124-130.

Thompson IL, Pauler DK, Goodman PJ, Tangen CM, Lucia MS, Parnes HL, Minasian LM, Ford LG, Lippman SM, Crawford ED, Crowley JJ, Coltman CA. 2004.Prevalence of prostate cancer among men with a prostate-specific antigen level $4.0 \mathrm{ng}$ per millilitre. $N$. Engl. J. Med., 350(22): 2239-2246.

Vickers AJ, Wolters T, Savage CJ, Cronin AM, O'Brien MF, Pettersson K, Roobol MJ, Aus G, Scardino PT, Hugosson J, Schröder FH, Lilja H. 2009. Prostate specific antigen velocity for early detection of prostate cancer: result from a large, representative, population-based cohort. Eur. Urol., 56(5): 753-760. 